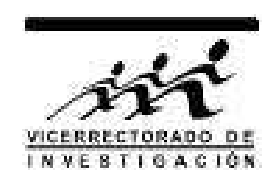

\title{
Aplicación de los modelos estocásticos en el dimensionamiento de las presas
}

\author{
Saúl Horqque Ferro*1,2 \\ ${ }^{1}$ Facultad de Ciencias Físicas, Universidad Nacional Mayor de San Marcos, Lima, Perú \\ ${ }^{2}$ Wharton Ingenieros SAC, Plaza Ricardo Palma 175-207, Lima, Perú
}

Recibido 26 noviembre 2011 - Aceptado 30 diciembre 2011

\begin{abstract}
Las técnicas de generación de muestras sintéticas proporcionada por la hidrología estocástica han demostrado ser una poderosa herramienta para la aplicación avanzada de procesos estocásticos en hidrología y en el tratamiento de los problemas relacionados con el dimensionamiento de las presas de almacenamiento, principalmente en las zonas con escasa información hidrológica; así como con la planificación y la operación de sistemas de aprovechamiento del recurso agua. En efecto, la hidrología estocástica genera sintéticamente series hidrológicas basado en el análisis de la estructura de los registros cortos, que permite contar con un amplio panorama de las posibilidades de ocurrencia de la disponibilidad hidráulica en el futuro, que a su vez posibilita con un grado de confiabilidad superior a los métodos tradicionales, determinar las dimensiones principales de la presa.

En este trabajo se presenta una metodología que permite mostrar la generación sintética de datos tomando como base las muestras históricas existentes y con ellas efectuar la simulación del vaso para determinar los niveles de operación apropiados y a la vez la altura óptima de la presa considerando la demanda requerida y la disponibilidad hidráulica.
\end{abstract}

Palabras claves: Hidrología estocástica, generación de muestras sintéticas, series de tiempo.

\section{Applications of stochastic models in the storage dams sizing}

The techniques for generating synthetic samples provided by stochastic hydrology have proved to be a powerful tool for advanced application of stochastic processes in hydrology and treatment of problems related to the size of the storage dams, mainly in areas with limited information hydrological as well as the planning and operation of water resource utilization. Indeed, stochastic hydrology allows the generation synthetic hydrologic series based on the analysis of the structure of short records, thus providing a panorama of possibilities of occurrence with hydro availability in the future, which allows a degree reliability than traditional methods, determining the main dimensions of the dam.

This paper presents a methodology to show the synthetic data generation based on existing historical samples and with them running a simulation of the vessel to determine appropriate levels of operation while the optimum height of the dam considering the required demand and the availability of hydropower.

Keywords: Stochastic Hydrology, generation of synthetic samples, time series.

La problemática actual por la atraviesa la humanidad, en relación al agua, es su escasez como recurso aprovechable, debido a la creciente falta para satisfacer sus necesidades básicas, de desarrollo y por pérdida de calidad por contaminación, principalmente; problemática que se agudizará cada vez más. Estimaciones recientes sugieren que el cambio paulatino del ecosistema mundial y regional reflejado en el cambio climático será responsable de alrededor del $20 \%$ del incremento de la escasez global y sus consiguientes consecuen-

*shorqque@hotmail.com cias 1], señalándose dentro de las principales amenazas al abastecimiento del recurso agua, las dificultades en la planificación de los recursos hidráulicos y las limitadas capacidades técnicas para la formulación y el desarrollo adecuado de las obras hidráulicas.

En el contexto de la actual problemática mundial en torno al agua, existe la creciente necesidad de difundir los conocimientos y las técnicas que permitan lograr los proyectos de aprovechamientos hidráulicos seguros, eficientes y económicos. Por ello, la escasez del agua co- 
mo recurso aprovechable, la poca o nula disponibilidad de la información hidrometeorológica y las características inherentes de los proyectos de aprovechamientos hidráulicos como, de larga maduración, alto costo inicial y fundamentalmente de ser de naturaleza irreversible, plantean optimizar los procesos de diseño hidráulico en todas las etapas inciales de su desarrollo.

En la solución de los problemas de aprovechamientos hidráulicos las herramientas utilizadas son los modelos matemáticos y los hidráulicos, modelos físicos, los primeros consisten en desarrollos teóricos y analíticos, y los segundos a través de resultados experimentales. En los problemas hidrológicos las soluciones se obtienen mediante modelos matemáticos de simulación hidrológica. Uno de los problemas a resolver en el diseño de las presas es la determinación del volumen de almacenamiento para que el embalse sea capaz de suministrar la demanda con un nivel de confiabilidad aceptable, haciendo que las dimensiones sean las óptimas.

En la estimación del volumen útil se han utilizado y se siguen utilizando -en primer lugar-, los métodos tradicionales, entre estos, el Diagrama de Rippl y el Algoritmo de Pico Secuente; en segundo lugar, se introduce la simulación del funcionamiento del vaso para un período largo, tomando en cuenta las variaciones mensuales, anuales y de todos los factores que intervienen en el agua almacenada en un determinado instante. Autores, como Yevjevich [2] cita además de lo anteriormente señalado, que consideramos el tercer lugar, el método experimental o generación de información sintética. Este método, consiste en la aplicación avanzada de procesos estocásticos para resolver el problema de embalses, por medio de series de caudales generadas estocásticamente que se combinan con variables relacionadas con las capacidades de un embalse.

La principal ventaja de este último método es que al obtener una amplia gama de capacidad de almacenamiento, permite una mayor flexibilidad en la toma de decisiones y se puede optimizar dicha capacidad mediante la evaluación económica que maximice los beneficios del proyecto estudiado a través del análisis llamado capacidad óptima del embalse.

\section{Modelación estocástica en series de tiempo}

\subsection{Características de los modelos estocás- ticos}

El modelamiento de series de tiempo son técnicas y procedimientos para estimar los modelos y sus parámetros a partir de los datos disponibles, son elaborados para reproducir o asemejar las características estadísticas más importantes de las series históricas. Como componentes de las series hidrológicas, podemos mencionar:

Tendencia. Componente determinística y transitoria de la serie, que definen una tendencia (ascendente o descendente).

Componente cíclica. Significa que las características estadísticas cambian anual o periódicamente.

Persistencia o componente autorregresiva. Es función de los parámetros estadísticos de la muestra. Se dice que los fenómenos hidrológicos tienen memoria.

Componente aleatoria o componente estocástica. Es función de los factores imprevisibles.

Estas componentes pueden ser reflejadas mediante las relaciones matemáticas de distintas formas. Por ejemplo,

$$
Z_{t}=\phi Z_{t-1}+\varepsilon_{t}
$$

\subsection{Proceso de modelamiento estocástico}

Una aproximación sistemática del proceso de modelamiento estocástico de series hidrológicas en el tiempo es presentada por Salas [3] et al., que consiste en los procedimientos que continuación se detallan brevemente.

\subsubsection{Identificación de la composición del mo- delo}

Esta fase consiste en decidir si el modelo será univariado, multivariado (anual o periódica) y modelos de desagregación o una combinación de estos.

\subsubsection{Selección del tipo de modelo}

Una vez identificada la composición, se puede seleccionar el tipo de modelo a usarse. Las distintas alternativas de modelos por las que se puede optar se encuentran detalladas por Salas et al. [3], entre estos: Modelos: Autorregresivos AR $(p)$, Autorregresivos con Promedios Móviles $\operatorname{ARMA}(p, q)$ y $\operatorname{PARMA}(p, q)$, Autorregresivos con Promedios Móviles Integrados $\operatorname{ARIMA}(p, q, r), \mathrm{Au}-$ torregresivo Multivariado de Desagregación (espacial y temporal: $\operatorname{MARMA}(p, q), \operatorname{MPARMA}(p, q)$, Modelos mixtos SARIMA, etc.

\subsubsection{Identificación de la forma del modelo}

Determinación del orden del modelo seleccionado (modelos de primer, segundo o tercer orden y pueden ser de parámetros constantes o periódicos) $(q, p)$. Así, tenemos como ejemplo, los modelos de parámetros cons- 
tantes:

$$
\begin{aligned}
\text { Modelo } \operatorname{AR}(q) & Z_{t}=\phi Z_{t-1}+\ldots+\phi Z_{t-p}+\varepsilon_{t} \\
\text { Modelo } \operatorname{AR}(1) & Z_{t}=\phi Z_{t-1}+\varepsilon_{t} \\
\text { Modelo } \operatorname{AR}(2) & Z_{t}=\phi Z_{t-1}+\phi_{1} Z_{t-2}+\varepsilon_{t} \\
\text { Modelo } \operatorname{ARMA}(p, q) & Z_{t}=\phi_{1} Z_{t-1}+\phi_{2} Z_{t-2}+\ldots \\
+\varepsilon_{t}-\theta_{1} \varepsilon_{t-1}-\ldots-\theta_{p} \varepsilon_{t-p} &
\end{aligned}
$$

De manera similar para los modelos autorregresivos con parámetros periódicos, se utiliza los modelos respectivos.

\subsubsection{Estimación de los parámetros del mode- lo}

Existen numerosas técnicas para determinar estos parámetros (la máxima verosimilitud y el de mínimos cuadrados).

\subsubsection{Pruebas de bondad de ajuste del modelo}

Se verifica si el modelo cumple con ciertos rangos de aplicación del modelo y reproduce las características estadísticas de interés. Dentro de éstas se considera la verificación de la independencia y normalidad de los residuos del modelo.

\subsubsection{Evaluación de la incertidumbre}

La incertidumbre del modelo puede ser evaluada comparando si existe diferencias en las estadísticas (la media, la varianza, el coeficiente de asimetría) generadas con otros modelos alternativos.

\section{Generación sintética de muestras y simulación del vaso}

Las series de las sucesivas etapas de cálculo, se denotan mediante la nomenclatura donde $X_{t}$ es para la serie histórica, $Y_{t}$ para la serie normalizada y $Z_{t}$ para la serie estandarizada.

\subsection{Cálculo de los principales estadísticos de la muestra}

\subsubsection{Serie de tiempo anuales}

Se calcula los principales estadísticos de la muestra como son la media de la serie, la varianza, el coeficiente de asimetría y la función de autocovarianza.

La función de autocovarianza mide el grado de la autodependencia lineal de la serie de tiempo. La autocovarianza $C_{k}$ entre $X_{t}$ y $X_{t+k}$ puede determinarse para la serie de tiempo histórica, $\left\{X_{t}\right\}$, donde $t=1,2, \ldots, N$, mediante

$$
C_{k}=\frac{1}{N} \sum_{t=1}^{N-k}\left(X_{t}-\bar{X}\right)\left(X_{t+k}-\bar{X}\right), 0 \leq k<N,
$$

donde $C_{k}$ es la autocovarianza de retraso $k, k$ es el tiempo de retraso o la distancia entre los pares $X_{t}$ y $X_{t+k}$. Una medida adimensional de la dependencia lineal, obtenida mediante el coeficiente de autocorrelación de retraso $k$ está dado por

$$
r_{k}=\frac{C_{k}}{C_{0}}=\frac{\sum_{t=1}^{N-k}\left(X_{t}-\bar{X}\right)\left(X_{t+k}-\bar{X}\right)}{\sum_{t=1}^{N}\left(X_{t}-\bar{X}\right)^{2}},
$$

donde $r_{k}$ es el coeficiente de autocorrelación de retraso $k, C_{0}$ es el coeficiente de autocorrelación para $k=0 \mathrm{y}$ la relación de $r_{k}$ y $k$ se denomina correlograma.

En las series de tiempo periódicas $\left\{X_{\nu, \tau}\right\}, \nu$ denota el año, $\tau$ el intervalo de tiempo dentro del año, $w$ el número de intervalos en el año (meses). Se calculan la media de la serie periódica, la varianza, el coeficiente de asimetría y el coeficiente de autocorrelación que se define como

$$
r_{\nu, \tau}=\frac{\frac{1}{N} \sum_{\nu=1}^{N}\left(X_{\nu, \tau}-\bar{X}\right)\left(X_{\nu, \tau-k}-\bar{X}_{\tau-k}\right)}{S_{\tau} \cdot S_{\tau-k}}
$$

cuando $\tau-k<1$, los términos de la ecuación (4) $1 / N$, $\nu=1, X_{\nu, \tau-k}$ y $\bar{X}_{\tau-k}$ son reemplazados por $1 /(N-1)$, $\nu=2, X_{\nu-1, w+\tau-k}$ y $X_{\nu-1, w+\tau-k}$, respectivamente.

\subsection{Verificación de la normalidad}

La muestra es normal para $g=0$, coeficiente de asimetría; $k=3$, coeficiente de curtosis. En caso contrario se emplean las técnicas de normalización Box-Cox y Box-Cox de doble potencia.

\subsection{Verificación de independencia}

Cuando el proceso estocástico se analiza en el dominio del tiempo, la verificación de la normalidad se realiza a través del correlograma. Según Anderson [5], los valores calculados deben estar fluctuando alrededor de cero entre ciertos límites de confianza,

$$
\begin{aligned}
& r_{k}(95 \%)=\frac{-1 \pm 1.645 \sqrt{N-k-1}}{N-k}, \\
& r_{k}(99 \%)=\frac{-1 \pm 2.326 \sqrt{N-k-1}}{N-k} .
\end{aligned}
$$




\subsubsection{Criterios de la decisión}

Si el $90 \%$ o más de los valores calculados en el correlograma caen dentro de los límites de confianza significa que la serie es independiente, esto es, define también la independencia de $\varepsilon t$.

Si menos del $90 \%$ de los valores calculados en el correlograma caen dentro de los límites de confianza la serie es dependiente, entonces se concluye que el modelo markoviano de primer orden no es adecuado para esta serie. Luego se prosigue usando los modelos de Markov de orden superior.

\subsection{Estandarización de la muestra}

Se utilizan las relaciones conocidas para las series periódicas y no periódicas, entre las cuales tenemos a la serie de variable normal estandarizada apropiado para las series mensuales, la serie de variable estandarizada por diferencia apropiado para modelos AR y la serie de variable modulada estandarizada para series anuales.

\subsection{Cálculo de los parámetros del modelo}

2.5.1. Propiedades de los modelos autorregresivos AR con parámetros constantes

i) Los parámetros $\mu, \sigma^{2}$ y $\sigma_{\varepsilon}^{2}$ son estimados a través de los datos históricos mediante la relación

$$
\sigma_{\varepsilon}^{2}=\sigma^{2}\left(1-\sum_{j=1}^{p} \phi_{j} \rho_{j}\right.
$$

donde $\phi_{j}$ es el coeficiente de autocorrelación, $\rho_{j}$ es el coeficiente de correlación de la variables $Y_{t}$ para un retraso $j$. Debemos considerar que $\phi_{j}=r_{j}$.

Para un modelo AR(1) y AR(2) la ecuación (7) queda simplificada en

$$
\begin{gathered}
\sigma_{\varepsilon}^{2}=\sigma^{2}\left(1-\phi^{2}\right) \quad \text { y en } \\
\sigma_{\varepsilon}^{2}=\sigma^{2} \frac{1+\phi_{2}}{1-\phi_{2}}\left[\left(1-\phi_{2}\right)^{2}-\phi_{1}^{2}\right],
\end{gathered}
$$

respectivamente. Finalmente, los parámetros $\sigma_{\varepsilon}^{2}$ para las series insesgadas se estiman usando la relación anterior multiplicada por el factor $N /(N-p)$.

ii) La determinación de los parámetros de autocorrelación. Se realiza a través de la función de autocorrelación serial. Para estimar los parámetros de los modelos $\operatorname{AR}(p)$, tenemos la ecuación de Yule-Walker [6] dado por

$$
\rho_{k}=\sum_{j=1}^{p} \phi_{j} \rho_{k-j}
$$

aquí es importante conocer la forma de $\rho_{k}\left(r_{k}\right)$ para obtener los parámetros del modelo $\operatorname{AR}(p)$, de modo que se define

$$
r_{k}=\phi_{1} r_{k-1}+\phi_{2} r_{k-2}+\ldots+\phi_{p} r_{k-p}, k>0 .
$$

De esta relación se obtienen para AR (1) los siguientes parámetros $r_{k}=\phi_{1}$ para $k>0, r_{0}=1$ y $r_{-1}=r_{1}$. Las soluciones para $\operatorname{AR}(2)$ se encuentran explicitadas en [3].

iii) Condiciones que deben cumplir los parámetros

\section{Estacionariedad}

De acuerdo a Yevjevich [2], para que un modelo autorregresivo $\operatorname{AR}(p)$ de parámetros constantes sea estacionario, se requiere que el conjunto de parámetros $\phi_{1}$, $\phi_{2}, \ldots, \phi_{p}$ esté dentro del círculo unitario y debe satisfacer la raíz de la ecuación característica dado por

$$
u^{p}-\phi_{1} u^{p-1}-\phi_{2} u^{p-2}-\ldots-\phi_{p}=0,
$$

donde $\left|u_{i}\right|<1$ para $i=1,2, \ldots, p$, siendo $u_{i}$ la raíz de la solución de la ecuación (12).

\subsubsection{Propiedades de los modelos AR con pa- rámetros periódicos}

i) Valor esperado y varianza de los modelos periódicos $\operatorname{AR}(p)$

$$
\begin{aligned}
E\left(Y_{\nu, \tau}\right) & =\mu_{\tau} \quad \tau=1,2, \ldots, w . \\
E\left(Z_{\nu, \tau}\right) & =E\left(\xi_{\nu, \tau}\right)=0 \quad \tau=1,2, \ldots, w . \\
\operatorname{Var}\left(Y_{\nu, \tau}\right) & =\sigma_{\tau}^{2} \quad \tau=1,2, \ldots, w . \\
\operatorname{Var}\left(Z_{\nu, \tau}\right) & =\operatorname{Var}\left(\xi_{\nu, \tau}\right)=1 \quad \tau=1,2, \ldots, w .
\end{aligned}
$$

ii) Determinación de la varianza de los residuales

$$
\sigma_{\varepsilon \tau}^{2}=1-\sum_{j=1}^{p} \phi_{j, \tau} \rho_{j, \tau} \quad \tau=1,2, \ldots, w .
$$

En particular, para el modelo $\mathrm{AR}(1)$ y $\mathrm{AR}(2)$ la ecuación (13) se simplifica como

$$
\begin{gathered}
\sigma_{\varepsilon \tau}^{2}=1-\phi_{1, \tau}^{2} \quad \mathrm{y} \\
\sigma_{\varepsilon \tau}^{2}=1-\phi_{1, \tau}^{2}-\phi_{2, \tau}^{2},
\end{gathered}
$$

respectivamente.

\subsubsection{Cálculo de los residuales}

Verificación de la normalidad e independencia i) De la estructura de los modelos AR, se calcula el valor de los residuales mediante la relación

$$
\varepsilon_{t}=Z_{t}-\phi_{1} Z_{t-1}-\phi_{2} Z_{t-2}-\ldots-\phi_{p} Z_{t-p}
$$

ii) Determinación de la varianza de los residuales. iii) Verificación de la independencia de los residuales. Se puede verificar mediante el correlograma y los límites de Anderson descritos. También se efectúa la prueba de Porte Manteau, que sirve para verificar en forma global la independencia de la muestra. 


\subsubsection{Criterio de la selección de los modelos}

Criterio de información de Akaike (AIC). Considera el Principio de Parsimonia en la construcción de modelos, fue sugerido inicialmente para seleccionar el modelo correcto entre los ARMA en competencia [3.

$$
\operatorname{AIC}(p, q)=N \ln \left[\sigma_{\varepsilon}^{2}\right]+2(p+q)
$$

donde $N$ es el tamaño de la muestra y $\sigma_{\varepsilon}^{2}$ es la varianza de los residuales.

Bajo este criterio el modelo seleccionado será el que tenga un AIC menor

\subsection{Generación de las muestras sintéticas}

La generación de números aleatorios con distribución normal estándar. Se reduce al cálculo del componente estocástico $\varepsilon=\xi \sigma_{\varepsilon}$ (ruido aleatorio).

Debe ser independiente y uniformemente distribuido en el intervalo $(0,1)$.

La generación de números aleatorios puede hacerse en parejas con la ecuación de Box-Muller [7,

$$
\begin{aligned}
\xi_{i} & =\sqrt{2 \ln \left[\frac{1}{u_{1}}\right]} \cos \left[2 \pi u_{2}\right] \\
\xi_{i+1} & =\sqrt{2 \ln \left[\frac{1}{u_{1}}\right]} \operatorname{sen}\left[2 \pi u_{2}\right]
\end{aligned}
$$

donde $\xi_{i}, \xi_{i+1}$ son los números aleatorios de normal estándar, con media cero, mientras que $u_{1}, u_{2}$ son los números aleatorios con distribución uniforme entre $(0,1)$.

\subsection{Proceso de desestandarización y des- normalización de los valores calculados}

Es el proceso inverso a lo cálculos de normalización y estandarización para obtener la muestra generada.

\subsection{Simulación del funcionamiento del va- so}

\subsubsection{Dimensionamiento de la presa}

Se requiere conocer las características principales del sistema y asumir ciertas dimensiones como asumir la altura, elevación desde el fondo, y las demás obras que comprende el sistema hidráulico, la política general del uso del agua, los alcances globales del proyecto, la necesidad de uno o más embalses en un sistema de aprovechamiento, la base para un análisis económico financiero del proyecto.

\subsubsection{Ecuación general de almacenamiento}

i) Variables de Entrada.

- Entradas por cuenca propia (IPC): a) Registros históricos, b) Registros sintéticos (son utilizados en las etapas avanzadas de estudio o diseño).

- Entradas por transferencia desde otras cuencas (IT).

- Entradas por volumen de lluvia en el vaso (VLL).

ii) Variables de extracción (VDEM).

- Por políticas de operación: extracción constante y/o extracción variable.

- Por pérdidas: evaporación (VEVA), infiltración (VINF), derrames (DERR).

iii) Intervalos de tiempo.

iv) Expresiones matemáticas de almacenamiento.

La ecuación general de almacenamiento está dado por

$$
V_{i+1}=V_{i}+X_{i}-S_{i}-P_{i} \quad, \quad V_{m} \leq V_{i+1} \leq V_{u},
$$

donde $X i=\mathrm{IPC}+\mathrm{IT}+\mathrm{VLL}, S i=$ VDEM, $P i=$ $\mathrm{VEVA}+\mathrm{VINF}+\mathrm{DERR}$ y $\Delta V=V_{i+1}-V_{i}$, y la ecuación general estocástica de almacenamiento se define como

$$
V_{i+1}=V_{i}+\left(X_{i}-Y_{i}\right)
$$

donde $V_{i}, V_{i+1}$ son los volúmenes almacenados al inicio $\mathrm{y}$ al final del intervalo, $X_{i}$ el el volumen total que ingresa al vaso durante el intervalo considerado e $Y_{i}=S_{i}+P_{i}$ es el volumen disminuído en el vaso. Cabe notar que tanto $X_{i}$ como $S_{i}$ son variables aleatorias.

\subsection{Procedimiento de la simulación}

Los cálculos para el método experimental se desarrollan en el orden que a continuación se detallan [4].

a) De la topografía del vaso se obtienen las relaciones elevación-área y elevación-volumen. Además se asume el NAMO y el NAMINO con los correspondientes volúmenes $\left(V_{u}, V_{m}\right)$.

b) Se inicia el cálculo a partir de un nivel inicial $h_{i}$, volumen inicial $V_{i}$ y un área de la superficie libre inicial $A_{i}$.

c) En una primera aproximación se calcula el volumen final del intervalo, con la relación

$$
V_{i+1}^{(1)}=V_{i}+X_{i}-S_{i}-P_{i} .
$$

d) Con el volumen obtenido en el paso anterior se obtienen los valores correspondientes de $h_{i+1}$ y $A_{i+1}$ y se calculan los valores medios $\bar{h}=0.5\left(h_{1}+h_{i+1}\right)$, $\bar{A}=0.5\left(A_{i}+A_{i+1}\right)$.

e) Se obtiene una nueva aproximación del volumen correspondiente al final del intervalo, $V_{i+1}^{(2)}=V_{i}+X_{i}-$ $S_{i}-P_{i}$. 
f) Si el nuevo volumen calculado $V_{i+1}^{(2)}$, es semejante al anterior $V_{i+1}^{(1)}$, se continua con el siguiente paso, sino se repite el proceso a partir del punto d) hasta que $V_{i+1}^{(k+1)} \cong V_{i+1}^{(k)}$.

Cuando del resultado del cálculo se obtiene un volumen $V_{i+1}$ mayor que $\mathrm{Vu}$, se registra un volumen derramado igual a la diferencia y se considera que $V_{i+1}=V u$. En cambio cuando $V_{i+1}$ es menor que $V_{m}$, se calcula el volumen de déficit igual a la diferencia y se considera que $V_{i+1}=V_{m}$.

g) Para un nuevo intervalo de tiempo $\Delta t$, se calculan las condiciones a partir del paso c).

\section{Aplicaciones}

El prototipo elegido para la aplicación de los modelos estocásticos en el diseño de presas, es la laguna La Victoria que tiene un sistema de aprovechamiento de agua en operación con una capacidad útil de $1.4 \mathrm{mi}-$ llones de $\mathrm{m}^{3}$ de agua y que forma parte del Sistema Hidráulico de afianzamiento de la Central Hidroeléctrica de Yaupi, localizada en la Región Central del Perú.

\subsection{Las características físicas principales de la cuenca}

Área de la cuenca (A), $4.479 \mathrm{~km}^{2}$; perímetro de la cuenca $(\mathrm{P}), 10.10 \mathrm{~km}$; pendiente medio de la cuenca $(\mathrm{S})$, 0.124 (determinado con el método Taylor Schwarz); ancho medio de la cuenca (Bm), $1.061 \mathrm{~km}$; tipo de corriente (aguas abajo de la laguna, es del tipo intermitente).

\subsection{Información hidrológica}

La zona de estudio cuenta con información hidrológica del tipo II, nula información de descargas y suficiente información de lluvia. Se consideró datos pluviométricos con una extensión de 40 años de registros.

\subsection{Análisis hidrológico}

i) Determinación de los coeficientes de escurrimiento periódicos (mensual), mediante la relación

$$
C_{\nu, \tau}=\frac{V_{d_{\nu, \tau}}}{V_{l l_{\nu, \tau}}} .
$$

ii) Determinación de los volúmenes con base a los datos de precipitación,

$$
V_{e \nu, \tau}=A \times C_{\nu, \tau} \times h_{l l_{\nu, \tau}} .
$$

\subsection{Generación de las muestras sintéticas}

\subsubsection{Proceso de la modelación}

Para el proceso de modelación se utilizó un modelo autorregresivo de parámetros periódicos para obtener las series en el orden descrito en la sección anterior, comenzando por, i) el cálculo de los principales estadísticos de la muestra histórica en forma periódica; ii) la verificación de la independencia de la muestra histórica, mediante los correlogramas y los límites permisibles; iii) se realizó el proceso de normalización; iv) la estandarización se efectúo con el criterio de la variable normal estandarizada, por ser la más apropiada para las series mensuales; v) el cálculo de los parámetros del modelo se realizaron para el modelo $\operatorname{PAR}(1)$ obteniéndose además el correlograma desarrollado, donde $r_{k}=\phi_{1}$; vi) la desviación estándar de los residuales se obtuvo a partir de la ecuación (14); vii) el cálculo de los residuales se obtuvieron a partir de la ecuación 1, con el detalle

$$
\begin{array}{ll}
\varepsilon_{\nu, 1}=Z_{\nu, 1}-\phi_{1,1} Z_{\nu-1,12} & \text { para el mes de enero, } \\
\varepsilon_{\nu, 1}=Z_{\nu, \tau}-\phi_{1, \tau} Z_{\nu, \tau-1} & \text { para los otros meses }
\end{array}
$$

la normalidad e independencia de los residuales fue verificado a través de los parámetros estadísticos; viii) se realizó la obtención de las muestras sintéticas; ix) para los parámetros de selección del modelo fue necesario calcular el coeficiente de parsimonia (PP20 215) y obtener el criterio de información de Akaike y finalmente, $\mathbf{x}$ ) se realizaron los cálculos de desestandarización y desnormalización para obtener la muestra sintética. También el proceso inverso a la estandarización y a la normalización.

\subsection{Simulación del funcionamiento del va- so}

Para la simulación del funcionamiento el vaso se i) definió el NAMINO y el NAMO del sistema, y la capacidad útil del vaso; ii) se verificó si la disponibilidad hidráulica de la cuenca satisface la demanda de $1.4 \mathrm{Mm}^{3}$ de agua, como inicialmente fue considerado; y iii) se definió los elementos de la simulación tales como los componentes físicos del sistema de aprovechamiento, presa de gravedad.

Las relaciones de la operación como la cuota de aporte asignada a esta laguna para satisfacer $1.4 \mathrm{Mm}^{3}$ al año dentro del período de estiaje (mayo-octubre), con un caudal dentro de un rango de $0.50 \mathrm{~m}^{3} / \mathrm{s}$ a $0.70 \mathrm{~m}^{3} / \mathrm{s}$. Las variables que se consideraron fueron las variables de estado como el volumen inicial y el final, el parámetro de diseño a través de la capacidad del embalse para diferentes alturas del muro y las variables de salida del modelo como los derrames y los déficits. 


\subsection{El proceso de la simulación}

\subsubsection{Determinación de las relaciones}

Se determinaron las relaciones elevación-volumen y elevación-área, calculando en base al plano topográfico y batimétrico mediante

$$
\begin{aligned}
& V=141.41 H^{1.1508} \\
& A=0.1449 H^{0.23794}
\end{aligned}
$$

donde $V$ se expresa en volúmenes de miles de $\mathrm{m}^{3}, H$ es la altura relativa en $\mathrm{m}, A$ es el área de la superficie del agua para cada nivel altimétrico en $\mathrm{km}^{2}$.

\subsubsection{Exploración de las diferentes condicio- nes}

Se establecen las condiciones iniciales tales como el nivel inicial, el nivel máximo de la operación, la extracción equivalente en $\mathrm{m}^{3} / \mathrm{s}$ y el mes de extracción.

Los resultados de la simulación obtenidos son el derrame total, el derrame promedio mensual, el número meses con derrame en el período, el derrame promedio mensual en el período, el derrame máximo, el derrame mínimo, los déficits totales, los déficits promedios mensuales, el número de meses con déficits en el período, los déficits máximo mensual, los déficits mínimo mensual.

\section{Discusión}

El proceso de generación de muestras sintéticas se realizó utilizando un modelo autorregresivo de de primer orden de parámetros periódicos PAR(1). Los resultados se resumen a continuación:

El anexo A muestra los volúmenes en miles de $\mathrm{m}^{3}$, resultado del análisis hidrológico, que consistió en desarrollar un modelo de descargas vs precipitación en base a la escasa información de descargas existentes (4 años), que permitió determinar el coeficiente de escurrimiento. Esta muestra constituye la base para la generación de muestras sintéticas.

En el anexo B, se presenta las estadísticas de la muestra histórica: la media, varianza, coeficiente de asimetría (g) y de curtosis (k), observándose que la muestra no es normal en ninguno de los meses. También en el mismo anexo, se observan los correlogramas mensuales, los cuales fluctúan dentro de los límites permisibles, determinándose que el $92 \%$ de la serie analizada cumple con los requisitos de independencia.

El anexo C] presenta la muestra normalizada utilizando el método de Box-Cox de primera potencia. Asimismo, los resultados del proceso de estandarización se han efectuado mediante la ecuación

$$
Z_{\nu, \tau}=\frac{Y_{\nu, \tau}-\bar{Y}_{\tau}}{\sigma_{\tau}}
$$

En el anexo D se observa los parámetros del modelo PAR(1), donde $r_{k}=\phi_{1}$ fue obtenida de los correlogramas desarrollados; la desviación estándar de los residuos fue obtenida a partir de la ecuación (14). Los valores obtenidos cumplen con las condiciones de estacionariedad del modelo.

El cálculo de los residuales, se efectuó usando la ecuación (11). Los valores del anexo E se consideran suficientes para ser considerados normal. Para la obtención de las muestras sintéticas, primero se generaron las muestras aleatorias con la distribución uniforme $(0,1)$, luego se calcularon los números aleatorios con la normal estándar ( $\xi_{1}$ y $\left.\xi_{2}\right)$, aplicando las ecuaciones de Box Müller y con ellos los residuales mediante la relación $\varepsilon=\xi \sigma_{\varepsilon}$. Basado en éstos valores se obtuvo el modelo autorregresivo de parámetros periódicos y en seguida los valores generados. Con los valores generados, se procedió con el proceso inverso de los cálculos de estandarización y normalización, para obtener la muestra sintética (anexo F).

Como se puede apreciar, los valores de la muestra histórica y los generados mediante el modelo aplicado son casi similares. Asimismo, en las estadísticas las diferencias no son significativas.

El anexo G muestra una parte de los resultados de la simulación del funcionamiento del vaso, sintetizado en tablas. La simulación fue realizada para distintos escenarios de operación, considerando las posibilidades de mayor o menor extracción (dentro del rango prefijado), con intervalo de tiempo mensual y bimensual, con los niveles de operación máximos y mínimos (altura de operación de la presa), con las pérdidas esperadas de agua por derrames y por supuesto con menores déficits de agua. También se observa en el proceso de simulación la influencia del mes de inicio de las operaciones. Resultando la mejor opción para la simulación la que considera como los datos de entrada para la extracción $0.66 \mathrm{~m}^{3} / \mathrm{s}\left(1.71 \times 10^{6} \mathrm{~m}^{3}\right.$ de agua $), N i v_{\max }$ con $10.5 \mathrm{~m}$, $N i v_{\min }$ con $2 \mathrm{~m}, V_{u}$ con $1.802 \times 10^{6} \mathrm{~m}^{3}$ de agua y con un tiempo de extracción de un mes de 30 días.

\section{Conclusiones}

Las técnicas de generación de muestras sintéticas a través del modelamiento estocástico, son de fácil acceso y han demostrado ser una herramienta bastante útil para determinar las dimensiones de las represas.

\section{Comentario final}

Este trabajo fue presentado en el III Congreso Nacional del Agua realizado en marzo del 2011, en la UNMSM, Lima, Perú. 
A. Volúmenes efectivos para la cuenca: $V_{e}=A \times C_{e} \times h_{l l} / 10^{3}$ miles de $\mathbf{m}^{3}$

\begin{tabular}{|c|c|c|c|c|c|c|c|c|c|c|c|c|}
\hline Año & Ene & Feb & Mar & Abr & May & Jun & Jul & Ago & Sep & Oct & Nov & Dic \\
\hline 1956 & 233.40 & 456.45 & 522.65 & 257.29 & 181.55 & 19.10 & 16.43 & 1.32 & 82.16 & 58.19 & 36.52 & 55.32 \\
\hline 1957 & 149.39 & 343.81 & 291.52 & 48.79 & 77.32 & 65.61 & 2.44 & 5.04 & 54.07 & 86.94 & 67.83 & 142.12 \\
\hline 1958 & 171.23 & 446.11 & 390.61 & 140.31 & 144.72 & 32.14 & 13.59 & 2.39 & 24.09 & 127.49 & 96.16 & 86.39 \\
\hline 1959 & 157.43 & 441.72 & 552.45 & 191.22 & 131.53 & 14.84 & 2.16 & 2.30 & 31.33 & 116.51 & 76.23 & 235.91 \\
\hline 1960 & 138.39 & 296.27 & 132.34 & 261.34 & 155.87 & 15.69 & 11.02 & 6.29 & 47.47 & 97.82 & 131.32 & 94.10 \\
\hline 1961 & 234.16 & 270.71 & 536.64 & 255.41 & 143.59 & 31.00 & 0.01 & 4.21 & 58.24 & 95.93 & 177.27 & 257.89 \\
\hline 1962 & 247.71 & 405.92 & 432.75 & 131.66 & 220.92 & 8.79 & 5.97 & 13.86 & 29.68 & 53.83 & 61.41 & 187.52 \\
\hline 1963 & 241.10 & 395.44 & 575.77 & 220.46 & 67.10 & 48.40 & 4.57 & 8.74 & 57.88 & 110.25 & 128.37 & 201.93 \\
\hline 1964 & 180.29 & 450.79 & 443.17 & 355.17 & 318.90 & 4.34 & 4.78 & 16.77 & 79.32 & 161.33 & 224.53 & 116.33 \\
\hline 1965 & 174.11 & 495.29 & 798.76 & 453.78 & 94.19 & 52.57 & 21.53 & 10.16 & 90.68 & 93.65 & 124.76 & 201.74 \\
\hline 1966 & 259.89 & 436.75 & 331.16 & 113.19 & 381.70 & 2.36 & 0.48 & 0.99 & 44.23 & 184.39 & 133.76 & 231.88 \\
\hline 1967 & 356.68 & 619.79 & 0 & & 149.43 & 38.67 & 32.23 & 17.10 & & & & 140.66 \\
\hline 1968 & 210.04 & 552.94 & 764.84 & 212.35 & 263.06 & 99.27 & 8.62 & 32.14 & 54.43 & 63.71 & 95.49 & 180.18 \\
\hline 1969 & 291.26 & 536.15 & 546.21 & 21084 & 72.72 & 173.38 & 25.80 & 7.43 & & 225.53 & & 255.98 \\
\hline 1970 & 334.42 & 488.52 & 327.31 & 345.16 & 496.44 & 202.12 & 55.88 & 13.49 & 150.57 & 190.30 & 272.63 & 161.10 \\
\hline 1971 & 285.08 & 436.01 & 664.03 & 22787 & 147.38 & 100.40 & 26.98 & 4.89 & 26.45 & 74.23 & & 224.09 \\
\hline 1972 & 308.90 & 307.26 & 601.66 & 181.95 & 201.48 & 86.03 & 4.13 & 0.00 & 0.00 & 111.17 & 70.91 & 158.89 \\
\hline 1973 & 326.30 & 641.49 & 3 & 8.97 & 72.10 & 48.22 & 18.96 & 21.85 & 39.04 & 56.71 & 132.12 & 386.72 \\
\hline 1974 & 215.84 & 395.23 & 292.20 & 305.29 & 13.30 & 165.45 & 23.30 & 9.53 & 39.16 & 69.90 & 90.31 & 223.82 \\
\hline 1975 & 228.75 & 566.44 & 415.22 & 150.60 & 272.57 & 91.70 & 7.46 & 10.62 & 93.00 & 75.91 & 83.62 & 198.74 \\
\hline 1976 & 340.47 & 352.93 & 2 & 232 & 44.49 & 4 & 9.98 & 7.5 & 55.73 & 44.28 & 58.53 & 125.39 \\
\hline 1977 & & & & & 229 & & 21. & 1. & & & & 148.41 \\
\hline 1978 & & 34 & & 1 & 158 & 56 & 11. & 5. & & 64 & & 93.95 \\
\hline 1979 & 1 & 41 & & 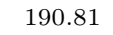 & 71 & 21 & 1 & 7. & & 68 & & 180.41 \\
\hline 1980 & 208.22 & 41 & 4 & 161.85 & 138.07 & 47. & 28.76 & 5.82 & 50 & 118.92 & 10 & 101.99 \\
\hline 1981 & 213.7 & & & 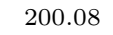 & 75. & 47. & 1.80 & 13. & & & & 241.79 \\
\hline 1982 & 217.95 & 469.87 & 3 & 218.07 & 24.55 & 56.72 & 16.36 & 7.51 & 50.88 & 131.57 & 283.97 & 190.70 \\
\hline 1983 & 248.43 & & & 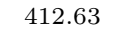 & 205.06 & & 9.08 & 22.79 & 125 & & & 442.23 \\
\hline 1984 & 344.07 & 612.15 & 7 & 434.09 & 251.60 & 92 & 19.41 & 11.15 & 75.96 & 224.24 & 271.93 & 410.04 \\
\hline 1985 & 349.78 & 255.18 & 445.99 & 517.23 & 122.22 & 208.46 & 98.14 & 31.58 & 99.98 & 78.75 & 152.85 & 191.82 \\
\hline 1986 & 274.24 & 883.3 & 1089.7 & 305.6 & 218.3 & 52.4 & 03.1 & 63. & 141 & 199.25 & 112 & 205.86 \\
\hline 1987 & 37 & 6 & & & 93 & & 3. & 5. & & & & 93.57 \\
\hline 1988 & 380.26 & 2 & 3 & 331.87 & 412.68 & 10.8 & 1.44 & 4.77 & 97.26 & 83.65 & 204.03 & 230.38 \\
\hline 1989 & 307.67 & & 101 & 774.48 & 38. & & 5.75 & 11. & & & 30 & 79.54 \\
\hline 1990 & 143. & 16 & & 53.15 & 1043.73 & 325.50 & 106.68 & 64.09 & 111.81 & 223.45 & 211.89 & 121.27 \\
\hline 1991 & 137.12 & & & & 143.70 & 110. & 20.40 & 3. & & 65.31 & 154 & 13.10 \\
\hline 1992 & 79.78 & 308. & & & 39.38 & 31. & 19.14 & 16.03 & 32 & 22.71 & 14.85 & 28.45 \\
\hline 1993 & 229.26 & & & & 167.33 & & 26.89 & 21.23 & & 85.02 & & 340.01 \\
\hline 199 & 230. & 56 & & & 117. & 66 & 4.94 & 1. & & 84. & & 70.11 \\
\hline 1995 & 107.88 & 563.71 & 778.18 & 108.69 & 60.45 & 195.40 & 9.60 & 1.32 & 37.20 & 132.96 & 177.84 & 198.34 \\
\hline Prom & 233.40 & 456.51 & 522.65 & 257.29 & 181.55 & 85.87 & 20.79 & 12.45 & 62.18 & 126.30 & 137.91 & 181.22 \\
\hline$\sigma$ & 81.290 & 138.403 & 217.411 & 146.792 & 176.459 & 83.439 & 26.110 & 14.330 & 39.851 & 61.373 & 74.286 & 96.259 \\
\hline$C_{V}$ & 0.348 & 0.303 & 0.416 & 0.571 & 0.972 & 0.972 & 1.256 & 1.151 & 0.641 & 0.486 & 0.539 & 0.531 \\
\hline
\end{tabular}

\section{B. Estadísticos de la muestra histórica}

\begin{tabular}{|c|c|c|c|c|c|c|c|c|c|c|c|c|}
\hline & Ene & Feb & Mar & Abr & May & Jun & Jul & Ago & Sep & Oct & Nov & Dic \\
\hline Med & 233.4 & 456.51 & 522.65 & 257.29 & 181.55 & 85.87 & 20.79 & 12.45 & 62.18 & 126.3 & 137.91 & 181.22 \\
\hline $\mathrm{S}$ & 81.29 & 138.4 & 217.41 & 146.79 & 176.46 & 83.44 & 26.11 & 14.33 & 39.85 & 61.37 & 74.29 & 96.26 \\
\hline $\mathrm{g}$ & 0.0775 & 0.5681 & 0.847 & 1.3042 & 3.282 & 1.6618 & 2.4571 & 2.5397 & 1.2774 & 0.5008 & 0.5905 & 0.8238 \\
\hline K & 2.33 & 4.2 & 3.56 & 5.7 & 17.19 & 5.52 & 8.75 & 10.12 & 4.9 & 2.36 & 2.75 & 4.11 \\
\hline
\end{tabular}




\section{Muestra normalizada}

\begin{tabular}{|c|c|c|c|c|c|c|c|c|c|c|c|c|}
\hline Año & Ene & Feb & Mar & Abr & May & Jun & Jul & Ago & Sep & Oct & Nov & Dic \\
\hline 1956 & 135.71 & 37.48 & 15.33 & 11.93 & 6.70 & 4.09 & 3.73 & 0.29 & 15.65 & 10.46 & 10.66 & 15.31 \\
\hline 1957 & 91.34 & 32.44 & 12.68 & 6.54 & 5.37 & 6.72 & 0.98 & 1.90 & 12.37 & 12.76 & 15.48 & 27.33 \\
\hline 1958 & 103.12 & 37.04 & 13.96 & 9.70 & 6.34 & 5.12 & 3.41 & 0.95 & 7.66 & 15.34 & 18.96 & 20.20 \\
\hline 1959 & 95.70 & 36.86 & 15.60 & 10.79 & 6.19 & 3.64 & 0.83 & 0.90 & 8.99 & 14.70 & 16.57 & 36.98 \\
\hline 1960 & 85.33 & 30.05 & 9.66 & 11.99 & 6.46 & 3.73 & 3.07 & 2.21 & 11.48 & 13.52 & 22.66 & 21.29 \\
\hline 1961 & 136.10 & 28.69 & 15.46 & 11.90 & 6.32 & 5.04 & -1.85 & 1.66 & 12.90 & 13.39 & 26.85 & 38.98 \\
\hline 1962 & 143.05 & 35.31 & 14.43 & 9.48 & 7.02 & 2.76 & 2.14 & 3.43 & 8.70 & 10.05 & 14.59 & 32.26 \\
\hline 1963 & 139.67 & 34.84 & 15.80 & 11.33 & 5.15 & 6.01 & 1.77 & 2.70 & 12.86 & 14.32 & 22.37 & 33.72 \\
\hline 1964 & 107.94 & 37.24 & 14.54 & 13.25 & 7.64 & 1.72 & 1.83 & 3.76 & 15.34 & 17.14 & 30.63 & 24.22 \\
\hline 1965 & 104.65 & 39.06 & 17.51 & 14.33 & 5.67 & 6.20 & 4.21 & 2.93 & 16.52 & 13.23 & 22.01 & 33.70 \\
\hline 1966 & 149.26 & 36.65 & 13.22 & 8.99 & 7.95 & 0.94 & -0.67 & -0.01 & 11.02 & 18.25 & 22.90 & 36.60 \\
\hline 1967 & 197.47 & 43.75 & 17.67 & 8.32 & 6.39 & 5.51 & 4.98 & 3.79 & 14.07 & 19.36 & 12.30 & 27.16 \\
\hline 1968 & 123.60 & 41.30 & 17.28 & 11.18 & 7.32 & 7.77 & 3.95 & 4.96 & 12.42 & 21.51 & 18.89 & 31.51 \\
\hline 1969 & 165.09 & 40.66 & 15.54 & 11.32 & 5.27 & 9.33 & 4.55 & 2.45 & 8.15 & 20.03 & 35.67 & 38.81 \\
\hline 1970 & 186.54 & 38.79 & 13.17 & 13.13 & 8.42 & 9.80 & 6.13 & 3.39 & 21.75 & 18.52 & 34.12 & 29.47 \\
\hline 1971 & 161.99 & 36.62 & 16.53 & 13.04 & 6.37 & 7.80 & 4.64 & 1.86 & 8.11 & 11.81 & 19.53 & 35.87 \\
\hline 1972 & 173.90 & 30.62 & 16.03 & 10.61 & 6.87 & 7.40 & 1.64 & -1.86 & -1.38 & 14.37 & 15.88 & 29.22 \\
\hline 1973 & 182.53 & 44.51 & 15.68 & 11.47 & 5.26 & 6.00 & 3.98 & 4.23 & 10.25 & 16.91 & 22.74 & 49.45 \\
\hline 1974 & 126.62 & 34.83 & 12.69 & 12.62 & 2.93 & 9.19 & 4.36 & 2.83 & 10.26 & 11.46 & 18.29 & 35.84 \\
\hline 1975 & 133.31 & 41.81 & 14.23 & 9.94 & 7.38 & 7.56 & 2.46 & 3.00 & 16.75 & 11.94 & 17.49 & 33.40 \\
\hline 1976 & 189.52 & 32.87 & 13.72 & 9.24 & 4.56 & 5.98 & 2.91 & 2.47 & 12.58 & 9.09 & 14.18 & 25.34 \\
\hline 1977 & 68.96 & 38.53 & 14.45 & 10.96 & 7.09 & 6.47 & 4.23 & 0.45 & 13.75 & 12.48 & 32.94 & 28.05 \\
\hline 1978 & 109.40 & 32.51 & 13.36 & 10.02 & 6.48 & 6.38 & 3.20 & 2.02 & 14.06 & 10.99 & 22.90 & 21.26 \\
\hline 1979 & 69.56 & 35.66 & 18.53 & 10.79 & 5.25 & 4.30 & 3.71 & 2.54 & 9.57 & 11.31 & 21.64 & 31.53 \\
\hline 1980 & 122.65 & 35.75 & 14.90 & 10.19 & 6.26 & 5.96 & 4.76 & 2.10 & 11.95 & 14.84 & 20.17 & 22.36 \\
\hline 1981 & 125.52 & 39.75 & 15.27 & 10.96 & 5.32 & 5.96 & 0.62 & 3.45 & 10.95 & 17.88 & 26.34 & 37.52 \\
\hline 1982 & 127.72 & 38.03 & 13.72 & 11.28 & 3.73 & 6.38 & 3.72 & 2.47 & 11.95 & 15.57 & 34.89 & 32.59 \\
\hline 1983 & 143.42 & 31.99 & 15.52 & 13.91 & 6.90 & 11.53 & 2.76 & 4.30 & 19.75 & 19.94 & 22.97 & 53.48 \\
\hline 1984 & 191.29 & 43.48 & 17.37 & 14.13 & 7.24 & 8.14 & 4.03 & 3.08 & 14.98 & 19.97 & 34.07 & 51.18 \\
\hline 1985 & 194.09 & 27.82 & 14.57 & 14.94 & 6.07 & 9.89 & 7.44 & 4.92 & 17.43 & 12.16 & 24.70 & 32.70 \\
\hline 1986 & 156.53 & 52.27 & 19.27 & 12.62 & 7.01 & 6.19 & 7.57 & 6.40 & 21.04 & 18.91 & 20.76 & 34.11 \\
\hline 1987 & 207.21 & 43.62 & 13.92 & 14.60 & 5.66 & 5.35 & 1.49 & 2.04 & 8.85 & 14.11 & 18.83 & 21.21 \\
\hline 1988 & 208.95 & 29.98 & 13.44 & 12.96 & 8.09 & 3.10 & 0.38 & 1.83 & 17.17 & 12.52 & 29.04 & 36.46 \\
\hline 1989 & 173.29 & 45.87 & 18.88 & 16.93 & 4.35 & 6.53 & 2.09 & 3.15 & 3.46 & 17.63 & 9.45 & 19.20 \\
\hline 1990 & 87.88 & 22.26 & 11.92 & 6.76 & 9.80 & 11.33 & 7.65 & 6.41 & 18.53 & 19.94 & 29.66 & 24.84 \\
\hline 1991 & 84.63 & 34.86 & 16.22 & 12.33 & 6.33 & 8.05 & 4.12 & 1.36 & 9.82 & 11.08 & 24.82 & 5.80 \\
\hline 1992 & 52.16 & 30.69 & 12.77 & 8.87 & 4.38 & 10.22 & 4.00 & 3.68 & 9.22 & 6.33 & 5.94 & 9.96 \\
\hline 1993 & 133.57 & 43.13 & 13.22 & 12.08 & 6.57 & 5.74 & 4.63 & 4.17 & 24.56 & 12.62 & 32.29 & 45.86 \\
\hline 1994 & 134.42 & 41.93 & 15.53 & 14.42 & 6.01 & 6.74 & 1.88 & 0.51 & 9.78 & 18.27 & 22.73 & 17.75 \\
\hline 1995 & 68.34 & 41.71 & 17.37 & 8.86 & 5.00 & 9.69 & 2.90 & 0.29 & 9.96 & 15.65 & 26.89 & 33.36 \\
\hline Med & 134.80 & 37.03 & 15.02 & 11.47 & 6.23 & 6.51 & 3.26 & 2.57 & 12.58 & 14.76 & 22.35 & 30.40 \\
\hline$\sigma$ & 42.0680 & 5.7941 & 2.0184 & 2.2260 & 1.3153 & 2.4543 & 2.0544 & 1.6936 & 4.9010 & 3.5961 & 7.2904 & 10.2369 \\
\hline Asim & -0.0003 & 0.0000 & 0.0002 & 0.0000 & 0.0000 & -0.0001 & -0.0005 & 0.0004 & 0.0000 & 0.0001 & -0.0009 & 0.0000 \\
\hline$\lambda$ & 0.8783 & 0.4811 & 0.2534 & 0.2477 & 0.0937 & 0.2112 & 0.1966 & 0.1946 & 0.4897 & 0.40862 & 0.5238 & 0.5648 \\
\hline
\end{tabular}

\section{Cálculo de parámetros}

Tabla : Modelos autoregresivo $\operatorname{PAR}(1)$ de parámetros periódicos

\begin{tabular}{|c|c|c|c|c|c|c|c|c|c|c|c|c|}
\hline & Ene & Feb & Mar & Abr & May & Jun & Jul & Ago & Sep & Oct & Nov & Dic \\
\hline$\phi_{1}$ & 0.517 & 0.264 & 0.599 & 0.34 & -0.171 & 0.338 & 0.473 & 0.808 & 0.53 & 0.164 & 0.338 & 0.379 \\
\hline$S_{y}$ & 42.0680 & 5.7941 & 2.0184 & 2.2260 & 1.3153 & 2.4543 & 2.0544 & 1.6936 & 4.9010 & 3.5961 & 7.2904 & 10.2369 \\
\hline$\sigma_{\varepsilon}$ & 0.8560 & 0.9645 & 0.8007 & 0.9404 & 0.9853 & 0.9411 & 0.8811 & 0.5892 & 0.8480 & 0.9865 & 0.9411 & 0.9254 \\
\hline $\mathrm{E}^{*}$ & $\mathrm{C}$ & $\mathrm{C}$ & $\mathrm{C}$ & $\mathrm{C}$ & $\mathrm{C}$ & $\mathrm{C}$ & $\mathrm{C}$ & $\mathrm{C}$ & $\mathrm{C}$ & $\mathrm{C}$ & $\mathrm{C}$ & C \\
\hline
\end{tabular}

* Estacionariedad.

C : Cumple.

Los parámetros deben estar dentro del círculo unitario. 
E. Cálculo de los residuales

\begin{tabular}{|c|c|c|c|c|c|c|c|c|c|c|c|c|}
\hline & Ene & Feb & Mar & Abr & May & Jun & Jul & Ago & Sep & Oct & Nov & Dic \\
\hline 1956 & 0 & 0 & 0 & 0 & 0 & 0 & 0 & 0 & 0 & 0 & 0 & 0 \\
\hline 1957 & -0.2711 & -0.5204 & -0.6877 & -1.8197 & -1.0335 & 0.3092 & -1.1504 & 0.4978 & 0.1679 & -0.5481 & -0.7546 & 0.0577 \\
\hline 1958 & -0.5984 & 0.2009 & -0.5303 & -0.6146 & -0.0529 & -0.5932 & 0.3422 & -1.0209 & -0.4956 & 0.3272 & -0.5189 & -0.8204 \\
\hline 1959 & -0.4145 & 0.2155 & 0.3031 & -0.3997 & -0.0841 & -1.1588 & -0.6273 & -0.0332 & -0.2095 & 0.1036 & -0.7865 & 0.9429 \\
\hline 1960 & -1.5082 & -0.8944 & 1.9346 & 1.1364 & 0.2130 & -1.1880 & 0.4422 & -0.1401 & -0.1111 & -0.3092 & 0.1605 & -0.9066 \\
\hline 1961 & 0.4911 & -1.4485 & 1.0770 & 0.1196 & 0.1063 & -0.6205 & -2.2042 & 1.4681 & 0.3522 & -0.3922 & 0.7462 & 0.6041 \\
\hline 1962 & -0.2372 & -0.3495 & -0.1174 & -0.7905 & 0.4533 & -1.7316 & 0.1794 & 0.9451 & -1.0602 & -1.1793 & -0.6209 & 0.5855 \\
\hline 1963 & 0.0214 & -0.4090 & 0.6138 & -0.1954 & -0.8265 & 0.0730 & -0.6274 & 0.6564 & 0.0184 & -0.1325 & 0.0454 & 0.3230 \\
\hline 1965 & -0.4047 & 0.5397 & 1.0234 & 0.8681 & -0.2069 & 0.0187 & 0.5261 & -0.1675 & 0.6931 & -0.5564 & 0.0978 & 0.3399 \\
\hline 1966 & 0.1769 & -0.1572 & -0.8526 & -0.8108 & 1.1223 & -2.7104 & -0.8409 & 0.0202 & 0.4898 & 1.0218 & -0.2515 & 0.5771 \\
\hline 1967 & 1.1764 & 0.7661 & 0.6167 & -1.8590 & -0.1199 & -0.4465 & 1.0318 & 0.0391 & -0.0774 & 1.2301 & -1.8104 & 0.2062 \\
\hline 1968 & -0.1029 & 0.8071 & 0.6758 & -0.5074 & 0.8057 & 0.2348 & 0.0958 & 1.1323 & -0.7790 & 1.8831 & -1.1091 & 0.2882 \\
\hline 1969 & 0.6640 & 0.4364 & 0.1179 & -0.1561 & -0.7363 & 1.3962 & 0.0856 & -0.5815 & -0.8651 & 1.6124 & 1.3323 & 0.1288 \\
\hline 1970 & 0.8053 & -0.0210 & -1.0995 & 1.0587 & 1.7936 & 0.7770 & 0.7662 & -0.6512 & 1.6174 & 0.7374 & 1.2618 & -0.7029 \\
\hline 1971 & 0.6932 & -0.2425 & 0.7903 & 0.4520 & 0.2257 & 0.4912 & 0.4227 & -0.9655 & -0.6876 & -0.6708 & -0.1095 & 0.6810 \\
\hline 1972 & 0.6532 & -1.3521 & 1.1597 & -0.5532 & 0.4244 & 0.1972 & -0.9592 & -1.9797 & -1.4621 & 0.3598 & -0.8410 & 0.2213 \\
\hline 1975 & -0.3104 & 0.8336 & -0.8844 & -0.5525 & 0.7559 & 0.1348 & -0.5884 & 0.5624 & 0.7181 & -0.9235 & -0.4011 & 0.5459 \\
\hline 1976 & 1.1490 & -1.0611 & -0.2179 & -0.7804 & -1.4411 & 0.2139 & -0.0670 & 0.0738 & 0.0339 & -1.5763 & -0.5868 & -0.0693 \\
\hline 1977 & -1.3098 & 0.6724 & -0.4375 & -0.1322 & 0.6153 & -0.2354 & 0.4823 & -1.6389 & 0.9039 & -0.6736 & 1.6677 & -0.7799 \\
\hline 1978 & -0.4854 & -0.6201 & -0.3573 & -0.3702 & 0.0825 & -0.1190 & -0.0027 & -0.3068 & 0.4760 & -1.0984 & 0.4312 & -0.9212 \\
\hline 1979 & -1.0896 & 0.1723 & 1.8784 & -0.8966 & -0.7946 & -0.6496 & 0.6450 & -0.1968 & -0.6039 & -0.8594 & 0.2278 & 0.1473 \\
\hline 1980 & -0.3459 & -0.1447 & 0.0711 & -0.5515 & -0.0718 & -0.2333 & 0.8378 & -0.8711 & 0.0189 & 0.0447 & -0.3067 & -0.6719 \\
\hline 1981 & 0.1853 & 0.5271 & -0.1575 & -0.2696 & -0.7272 & 0.0082 & -1.1758 & 1.5498 & -0.6044 & 0.9215 & 0.2553 & 0.4878 \\
\hline 1982 & -0.5280 & 0.2172 & -0.7486 & 0.1370 & -1.9115 & 0.5879 & 0.2537 & -0.2472 & -0.0963 & 0.2480 & 1.6447 & -0.4382 \\
\hline 1983 & 0.0940 & -0.9239 & 0.7677 & 1.0116 & 0.6994 & 1.8731 & -1.2083 & 1.2149 & 0.9213 & 1.2007 & -0.4013 & 2.2229 \\
\hline 1984 & 0.1768 & 0.7578 & 0.4961 & 0.8021 & 0.9753 & 0.4044 & 0.0608 & -0.0066 & 0.3322 & 1.3693 & 1.1181 & 1.4203 \\
\hline 1985 & 0.3600 & -1.9611 & 0.7258 & 1.6362 & 0.1465 & 1.4194 & 1.3871 & -0.2618 & 0.2551 & -0.8855 & 0.5671 & 0.1030 \\
\hline 1986 & 0.4000 & 2.4929 & 0.5291 & -0.1975 & 0.6795 & -0.3271 & 2.1598 & 0.5645 & 0.5282 & 0.8720 & -0.6084 & 0.4450 \\
\hline 1987 & 1.5338 & 0.6831 & -1.2286 & 1.5939 & -0.1939 & -0.3229 & -0.6386 & 0.3773 & -0.5922 & -0.0552 & -0.4213 & -0.7144 \\
\hline 1988 & 2.2265 & -1.6822 & -0.0576 & 0.9395 & 1.5317 & -1.8657 & -0.7452 & 0.6896 & 1.1710 & -0.7751 & 1.1287 & 0.2441 \\
\hline 1989 & 0.6087 & 1.2837 & 0.9984 & 1.8057 & -1.0099 & 0.4919 & -0.5722 & 0.7994 & -2.0411 & 1.1032 & -2.0386 & -0.4237 \\
\hline 1990 & -0.5497 & -2.2541 & -0.0103 & -1.5907 & 2.3509 & 1.0501 & 1.2098 & 0.5342 & 0.0137 & 1.2415 & 0.5166 & -0.9234 \\
\hline 1991 & -0.9117 & -0.0595 & 0.8151 & 0.1854 & 0.1404 & 0.6024 & 0.1220 & -1.0559 & -0.1834 & -0.9303 & 0.6850 & -2.5314 \\
\hline 1994 & -0.7900 & 0.8472 & -0.2560 & 1.2397 & 0.0616 & 0.1506 & 0.7155 & -0.6760 & 0.0749 & 1.0699 & -0.2770 & -1.2551 \\
\hline 1995 & -0.9413 & 1.2237 & 0.6801 & -1.5682 & -1.1332 & 1.6131 & -0.7882 & -1.2104 & 0.1809 & 0.3361 & 0.5400 & 0.0530 \\
\hline Med & 0.0032 & -0.0018 & -0.0026 & -0.0039 & -0.0099 & 0.0276 & -0.0174 & 0.0385 & -0.0335 & 0.0325 & 0.0300 & 0.0217 \\
\hline Asim & 0.4481 & -0.2158 & -0.1168 & -0.0004 & -0.1216 & -0.3924 & -0.0039 & -0.3070 & 0.0578 & -0.0742 & -0.0783 & -0.1343 \\
\hline
\end{tabular}

Los residuales se consideran normales e independientes. 
F. Muestras generadas que han sido desestandarizadas y desnormalizadas

\begin{tabular}{|c|c|c|c|c|c|c|c|c|c|c|c|c|}
\hline & Ene & Feb & Mar & Abr & May & Jun & Jul & Ago & Sep & Oct & Nov & Dic \\
\hline 1956 & 301.52 & 250.05 & 307.14 & 132.88 & 65.24 & 21.95 & 2.79 & 3.34 & 31.79 & 57.92 & 68.24 & 88.67 \\
\hline 1957 & 134.47 & 421.71 & 735.79 & 416.78 & 77.30 & 181.31 & 15.43 & 19.35 & 115.71 & 293.72 & 252.45 & 358.66 \\
\hline 1958 & 281.21 & 431.82 & 387.46 & 282.96 & 261.67 & 237.06 & 44.30 & 10.75 & 100.19 & 195.23 & 141.73 & 118.42 \\
\hline 1959 & 276.26 & 532.77 & 373.38 & 59.34 & 240.52 & 108.73 & 14.00 & 6.59 & 28.73 & 142.71 & 73.60 & 186.73 \\
\hline 1960 & 244.41 & 454.35 & 339.14 & 117.77 & 62.65 & 3.34 & 3.36 & 14.34 & 49.05 & 228.10 & 354.80 & 350.30 \\
\hline 1961 & 280.81 & 540.67 & 461.65 & 331.41 & 105.66 & 23.30 & 2.78 & 0.19 & 46.41 & 31.85 & 164.73 & 153.13 \\
\hline 1962 & 136.96 & 338.59 & 411.96 & 248.12 & 33.39 & 40.71 & 13.52 & 5.48 & 69.22 & 127.26 & 99.92 & 166.54 \\
\hline 1963 & 191.59 & 358.64 & 282.06 & 350.05 & 31.10 & 14.69 & 21.61 & 8.91 & 23.34 & 80.16 & 205.15 & 225.85 \\
\hline 1964 & 165.12 & 621.00 & 485.53 & 363.82 & 52.54 & 194.79 & 6.04 & 7.37 & 13.82 & 203.10 & 263.21 & 159.11 \\
\hline 1965 & 314.62 & 520.65 & 838.23 & 582.91 & 10.14 & 98.78 & 17.71 & 10.37 & 52.30 & 100.65 & 175.10 & 163.45 \\
\hline 1966 & 148.23 & 545.50 & 636.22 & 290.80 & 45.76 & 66.08 & 67.90 & 25.57 & 78.34 & 155.49 & 120.34 & 207.43 \\
\hline 1967 & 246.37 & 320.84 & 351.45 & 155.74 & 82.77 & 135.11 & 14.72 & 12.94 & 164.88 & 74.11 & 95.27 & 51.10 \\
\hline 1968 & 96.97 & 291.14 & 322.67 & 68.98 & 46.49 & 48.92 & 7.90 & 1.96 & 125.82 & 292.96 & 100.15 & 30.00 \\
\hline 1969 & 146.82 & 534.61 & 233.67 & 98.07 & 115.53 & 30.71 & 29.70 & 11.49 & 103.39 & 179.34 & 227.06 & 208.32 \\
\hline 1970 & 201.02 & 200.61 & 374.97 & 108.55 & 257.90 & 66.22 & 150.53 & 18.57 & 63.11 & 143.03 & 36.07 & 166.53 \\
\hline 1971 & 217.99 & 704.17 & 859.72 & 380.15 & 212.73 & 43.22 & 6.60 & 2.85 & 51.89 & 86.52 & 89.25 & 163.76 \\
\hline 1972 & 185.66 & 329.07 & 332.59 & 147.18 & 22.94 & 11.85 & 1.06 & 0.11 & 24.33 & 166.06 & 91.27 & 92.06 \\
\hline 1973 & 184.94 & 655.07 & 38.27 & 265.49 & 384.69 & 10.30 & 15.19 & 7.30 & 75.42 & 123.83 & 261.48 & 377.46 \\
\hline 1974 & 337.04 & 469.13 & 387.77 & 152.98 & 98.68 & 14.93 & 2.97 & 1.34 & 45.52 & 79.93 & 84.38 & 103.64 \\
\hline 1975 & 337.98 & 470.08 & 504.36 & 284.97 & 176.17 & 42.85 & 2.02 & 12.81 & 85.20 & 72.29 & 86.47 & 320.45 \\
\hline 1976 & 350.32 & 556.57 & 642.29 & 465.36 & 270.92 & 46.37 & 12.62 & 11.37 & 74.20 & 162.28 & 251.31 & 161.78 \\
\hline 1977 & 185.12 & 334.27 & 31.81 & 270.53 & 404.38 & 268.32 & 7.31 & 28.66 & 83.14 & 135.88 & 44.13 & 23.96 \\
\hline 1978 & 192.56 & 351.51 & 449.44 & 56.47 & 291.18 & 143.15 & 0.40 & 0.76 & 22.10 & 79.88 & 163.97 & 242.54 \\
\hline 1979 & 173.34 & 237.89 & 323.06 & 111.47 & 91.59 & 27.81 & 0.67 & 1.44 & 39.58 & 47.75 & 84.83 & 132.18 \\
\hline 1980 & 201.25 & 304.54 & 237.12 & 121.86 & 70.87 & 41.37 & 1.10 & 1.92 & 54.55 & 98.17 & 99.96 & 177.19 \\
\hline 1981 & 118.36 & 229.61 & 224.03 & 324.13 & 149.31 & 104.68 & 15.44 & 6.85 & 87.75 & 79.62 & 96.23 & 104.57 \\
\hline 1982 & 173.52 & 952.81 & 1035.31 & 437.79 & 226.08 & 77.42 & 8.45 & 6.28 & 99.46 & 137.25 & 171.47 & 210.71 \\
\hline 1983 & 270.57 & 526.63 & 690.86 & 218.31 & 233.04 & 13.99 & 3.81 & 3.69 & 21.55 & 103.92 & 125.72 & 240.78 \\
\hline 1984 & 239.62 & 451.77 & 234.05 & 114.66 & 45.07 & 42.94 & 12.92 & 7.53 & 37.25 & 228.60 & 169.90 & 85.91 \\
\hline 1985 & 127.24 & 316.22 & 267.82 & 184.22 & 64.24 & 47.30 & 6.21 & 3.59 & 30.24 & 118.13 & 182.17 & 325.43 \\
\hline 1986 & 225.02 & 644.46 & 666.65 & 234.69 & 399.44 & 23.13 & 24.69 & 11.24 & 75.99 & 96.53 & 82.73 & 135.38 \\
\hline 1987 & 166.96 & 322.76 & 373.19 & 228.47 & 99.41 & 20.56 & 11.49 & 5.02 & 96.86 & 180.66 & 95.37 & 60.54 \\
\hline 1988 & 136.59 & 592.38 & 618.12 & 291.92 & 208.00 & 25.35 & 3.07 & 1.54 & 8.71 & 221.08 & 278.10 & 191.73 \\
\hline 1989 & 230.82 & 585.92 & 916.52 & 953.15 & 96.11 & 45.43 & 61.28 & 4.96 & 76.26 & 152.40 & 78.31 & 350.86 \\
\hline 1990 & 249.71 & 575.40 & 842.31 & 823.30 & 3.15 & 7.85 & 0.61 & 0.35 & 41.91 & 222.42 & 241.21 & 282.55 \\
\hline 1991 & 288.01 & 676.14 & 567.32 & 206.51 & 75.11 & 6.66 & 3.94 & 2.70 & 28.18 & 187.20 & 182.73 & 252.32 \\
\hline 1992 & 270.34 & 270.00 & 178.29 & 172.50 & 120.04 & 17.01 & 0.13 & 0.35 & 27.25 & 112.88 & 110.17 & 68.53 \\
\hline 1993 & 152.75 & 514.69 & 409.95 & 128.40 & 158.06 & 55.18 & 20.45 & 4.02 & 79.59 & 149.23 & 121.28 & 113.50 \\
\hline 1994 & 36.67 & 379.40 & 208.22 & 4.38 & 65.91 & 76.12 & 5.71 & 3.28 & 44.24 & 93.36 & 145.44 & 65.42 \\
\hline 1995 & 203.36 & 322.77 & 384.73 & 193.58 & 37.75 & 15.77 & 5.67 & 4.05 & 51.33 & 240.60 & 144.73 & 60.61 \\
\hline Med & 210.55 & 453.41 & 474.13 & 261.02 & 161.34 & 70.53 & 18.15 & 7.28 & 60.71 & 144.55 & 149.01 & 174.45 \\
\hline$\sigma$ & 71.59 & 159.83 & 218.06 & 191.28 & 124.30 & 8.41 & 28.15 & 6.77 & 34.28 & 64.90 & 73.44 & 96.84 \\
\hline
\end{tabular}

\section{G. Simulación del vaso}

Se presenta algunos resultados de la simulación que permite observar el comportamiento del vaso para las dimensiones propuestas, con las muestras generadas. Para un volumen de extracción mensual $V_{\text {ext }}=1.814 \times 10^{6}$ $\mathrm{m}^{3}$ de agua $\left(0.70 \mathrm{~m}^{3} / \mathrm{s}\right)$, para una capacidad del vaso $V_{u t i l}=1.687 \times 10^{6} \mathrm{~m}^{3}$ de agua, para un nivel máximo Niv $v_{\max }=10 \mathrm{~m}$ y un nivel mínimo de $N i v_{\min }=2 \mathrm{~m}$.

\begin{tabular}{llll}
\hline Mes de extracción & $\begin{array}{l}\text { Derrame mensual es- } \\
\text { perado }\left(\text { miles de } \mathrm{m}^{3}\right)\end{array}$ & $\begin{array}{l}\text { Déficit mensual espe- } \\
\text { rado }\left(\text { miles de } \mathrm{m}^{3}\right)\end{array}$ & Tipo de extracción \\
\hline Abril & 48.47 & 2.81 & conveniente \\
Mayo & 50.13 & 4.51 & conveniente \\
Junio & 53.14 & 7.62 & no conveniente \\
Julio & 56.51 & 11.04 & no conveniente \\
Agosto & 56.95 & 11.52 & no conveniente \\
Setiembre & 52.91 & 7.34 & no conveniente \\
Octubre & 49.62 & 3.80 & conveniente \\
\hline
\end{tabular}




\section{Referencias}

[1] IV Foro Mundial del Agua, México 2006; Informe sobre el Desarrollo de los Recursos Hídricos, UNESCO (2006).

[2] V. Yevjevich, Stochastic Processes in Hidrology, Water Resources Publications, Fort Collins, Colorado (1972).

[3] J. D. Salas, J. W. Delleur, V. Yevjevich y W. L. Lane; Applied Modeling in Hidrologic Time Series, Water Resources Publications, Fort Collins, Colorado (1980).

[4] J. A. Maza, V. Franco; Técnicas experimentales, manual de diseño de obras civiles; Comisión Federal de Electricidad, Instituto de Investigaciones Eléctricas, México D.F. (1983).

[5] R. L. Anderson, Distribution of the Serial Correlation Coeficients, Annales Math. Statistics 13, 1 (1942).

[6] G. U. Yule, On a method of investigating periodicities in disturbed series wiht special reference to Wolfer's sunspot numbers, Phil. Trans. Royal Soc. A 226, 267 (1937).

[7] G. E. P. Box y M. E. Muller; A note on the generation of normal deviates, Ann. Math. Stat. 28, 610 (1958). 